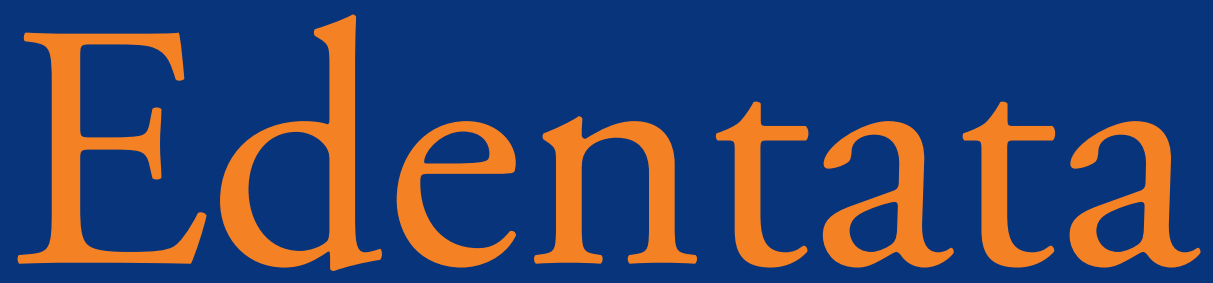

The Newsletter of the IUCN/SSC Anteater, Sloth and Armadillo Specialist Group

December 2014 • Number 15
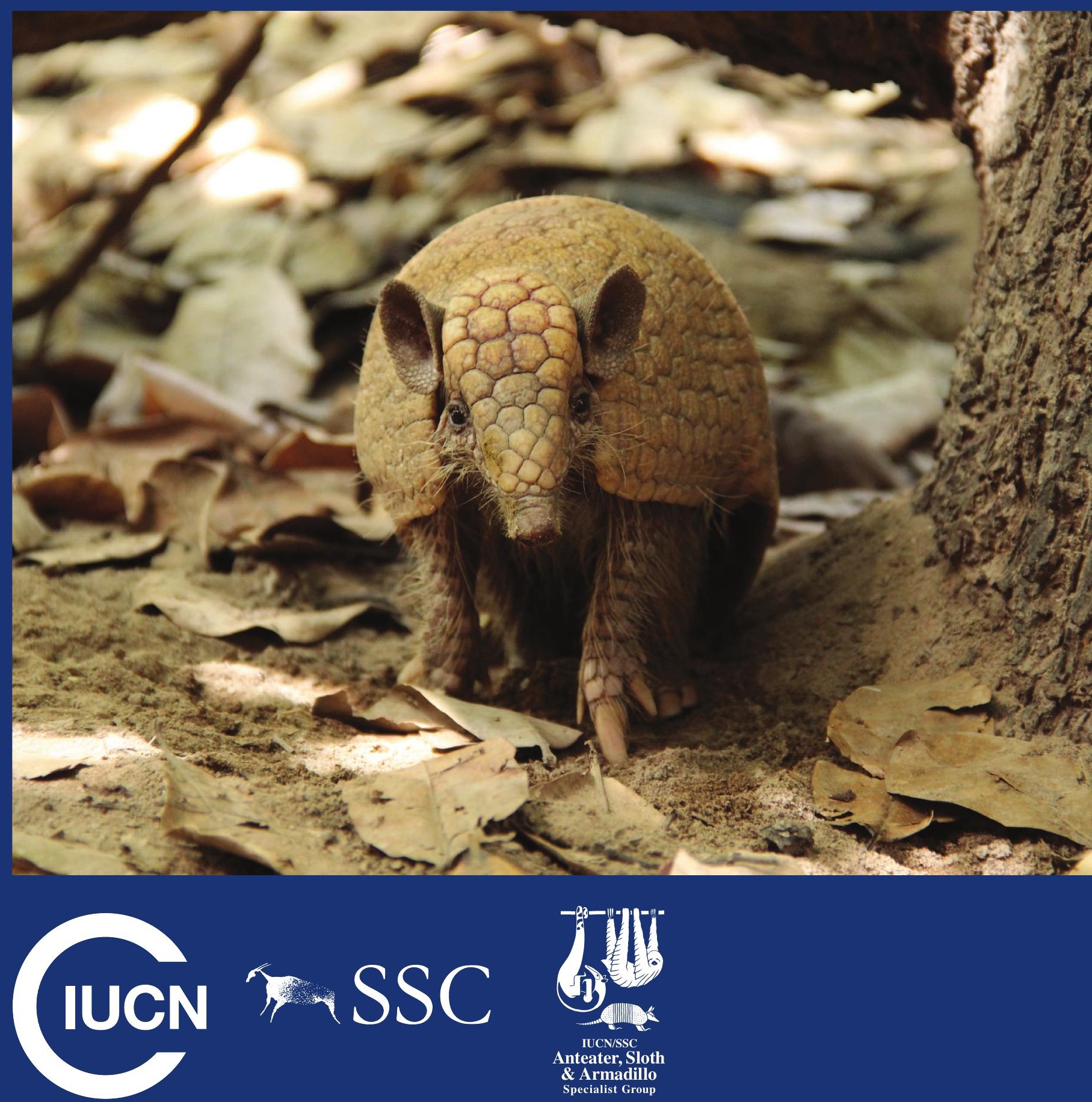

TS SSC

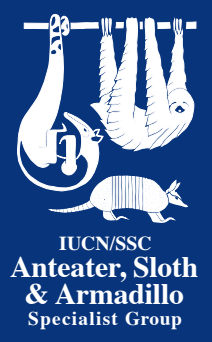




\title{
Respuesta antidepredatoria de osos hormigueros (Myrmecophaga tridactyla) mantenidos en cautividad
}

\author{
Cristian Gabriel Orlando ${ }^{\mathrm{A}, \mathrm{B}, \mathrm{C}, 1}$ y Gustavo Javier Fernández ${ }^{\mathrm{A}}$
}

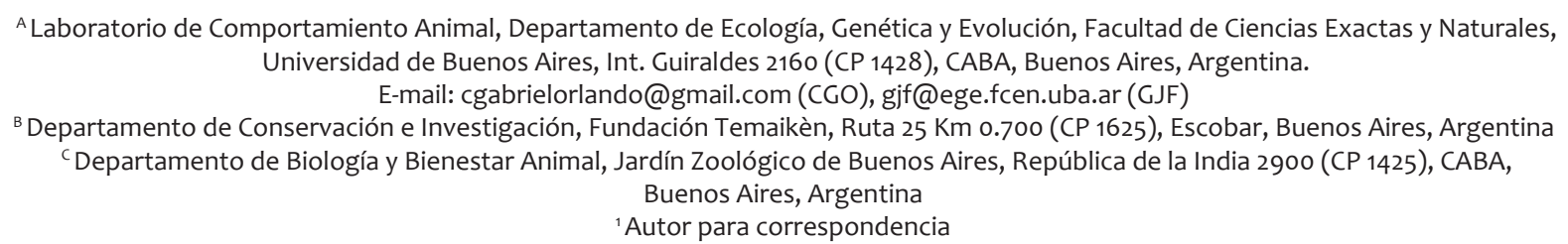

Resumen Muchos mamíferos son capaces de percibir y responder al riesgo de depredación a partir de señales emitidas por sus depredadores, aún en ausencia de una experiencia ecológica, evolutiva e incluso de ambas. Sin embargo, se ha propuesto que el cautiverio puede afectar dicho comportamiento debido probablemente a los costos que acarrean para las presas el conservar las conductas antidepredatorias. En el presente trabajo se analizó la respuesta de osos hormigueros (Myrmecophaga tridactyla) mantenidos en zoológicos ( $\mathrm{n}=7$ ) al ser expuestos a estímulos olfativos y auditivos de un depredador natural (yaguareté, Panthera onca). Para ello se comparó la respuesta comportamental que tenían frente a heces y vocalizaciones de yaguareté con aquella desplegada frente a señales de aguará guazú (Chrysocyon brachyurus), un carnívoro simpátrico no-depredador, y oveja (Ovis aries), una especie inocua alopátrica. Los resultados mostraron que los osos hormigueros en cautiverio conservan la capacidad de responder y discriminar entre los tratamientos, principalmente sobre la base de las vocalizaciones, pero que tras largos períodos de aislamiento las conductas antidepredatorias pueden verse alteradas, incompletas o incluso faltar. A pesar de que estos son resultados preliminares, el experimento apoya la hipótesis del mantenimiento de la respuesta antidepredatoria en los osos hormigueros mantenidos en cautividad que puede ser de suma importancia a la hora de definir estrategias para la reintroducción de animales criados en cautiverio y la reconstrucción de ecosistemas que incluyan poblaciones de esta especie.

Palabras clave: comportamiento en cautiverio, Panthera onca, reconocimiento de predadores, señales interespecíficas

\section{Antipredatory behavior of giant anteaters (Myrmecophaga tridactyla) in captivity}

Abstract Many mammals are able to perceive and respond to predation risk from signals produced by their predators, even in the absence of an ecological or evolutionary experience, or both. However, it has been proposed that captivity may affect such behavior, probably due to the unnecessary costs related to preserving the antipredatory responses in that condition. In the present work we analyzed the response of captive anteaters (Myrmecophaga tridactyla; $\mathrm{n}=7$ ) exposed to olfactory and acoustic stimuli from a natural predator (jaguar, Panthera onca). We compared the behavioral response of anteaters exposed to jaguar feces and vocalizations with those displayed when exposed to maned wolf (Chrysocyon brachyurus) and sheep (Ovis aries) signals. The maned wolf represents a sympatric carnivorous species that does not predate on anteaters, and the sheep represents an allopatric herbivorous non-predator species. The results showed that the anteaters in captivity retain the capability to respond to and discriminate between the different treatments, mainly when exposed to vocalizations. Despite this, the antipredatory behaviors may become altered, incomplete or even lacking after large periods of isolation. Even though these are preliminary results, the experiment supports the hypothesis that captive anteaters are able to retain some predator recognition ability, and this result could be relevant when defining strategies for the reintroduction of captive-reared animals and the restoration of ecosystems that hold populations of this species.

Keywords: behavior in captivity, interspecific signals, Panthera onca, predator recognition 


\section{INTRODUCCIÓN}

La depredación es una importante fuerza selectiva que conduce a diversas adaptaciones de las presas, no sólo a nivel morfológico, fisiológico o de historia de vida, sino también a nivel comportamental (Lima \& Dill, 1990; Endler, 1991). Dicha presión ha favorecido el desarrollo de diversos mecanismos en las presas para detectar depredadores y adoptar estrategias antidepredatorias antes de su ataque (Caro, 2005; Monclús et al., 2005). La evitación del depredador, sin embargo, suele ser costosa dado que afecta el tiempo y energía disponibles para actividades como la alimentación, apareamiento y defensa territorial a la vez que puede forzar a la presa a utilizar hábitats subóptimos (Brown \& Chivers, 2005). Es por ello que para responder adecuadamente minimizando los costos ante estímulos falsos, las presas deben ser capaces de reconocer específicamente al depredador (Anson \& Dickman, 2013).

El mantenimiento de comportamientos de evitación ante estímulos que pueden indicar cierto riesgo genera pérdidas de oportunidad cuando la presión de depredación es altamente variable (Anson \& Dickman, 2013). En este caso, es esperable que los mecanismos de reconocimiento mediante aprendizaje fueran la forma más extendida y económica de respuesta (Caro, 2005). Por otra parte, el aislamiento afectará la respuesta de las presas a estímulos de los depredadores, aunque el grado de pérdida de reconocimiento dependerá de factores tales como el grado en que el comportamiento antidepredatorio se basa en la experiencia (Coss, 1999; Blumstein, 2002), el tipo de señales que evocan dicho comportamiento (Blumstein et al., 2000), el costo que implica para la presa el expresarlo (Magurran, 1999; Berger et al., 2001), la letalidad del depredador (Brown, 1999), el número de generaciones que se encuentra bajo esta selección relajada (Hollén \& Manser, 2007) y la tasa de mutación de la especie (Blumstein et al., 2006).

El estudio de la capacidad de reconocimiento de depredadores presente en las presas es particularmente importante cuando se analiza la posibilidad de reintroducir animales criados o mantenidos en cautiverio a su hábitat natural (Curio, 1996; Caro, 2005). Parte del éxito de estas reintroducciones depende de la probabilidad de supervivencia de los mismos, y ésta, a su vez, obedece en gran parte a la capacidad que tengan las presas para responder a estímulos indicadores de riesgo de depredación en forma apropiada (Beck et al., 1991; Short et al., 1992; Miller et al., 1994).

El oso hormiguero (Myrmecophaga tridactyla) es uno de los mamíferos más distintivos de América Central y del Sur, extendiéndose desde el sur de Belice y Guatemala hasta el norte argentino (Wetzel, 1982). Sin embargo, se ha estimado una pérdida poblacional de al menos un 30\% en los últimos 10 años, en base a extinciones locales, modificación y pérdida de hábitat, y muertes causadas por incendios y accidentes de tránsito (IUCN, 2014). Esto se suma a la amenaza a largo plazo que de por sí sufre la especie debido a factores tales como la alta especialización en su dieta, la baja capacidad de fuga, la alta presión por caza y la baja tasa reproductiva seguida por el prolongado cuidado de su cría (Fonseca \& Aguiar, 2004). Por estos motivos, la especie se encuentra listada como Vulnerable (VU) a nivel internacional (IUCN, 2014) y a nivel nacional en Argentina (Superina et al., 2012), llevando a diferentes intentos por recuperar la especie y sus hábitats. Actualmente existe un importante plan de reintroducción de osos hormigueros en los Esteros del Iberá, Argentina (Pérez Jimenez, 2006). Este proyecto considera que entre los principales problemas para su desarrollo se encuentran la escasa disponibilidad de información sobre la ecología de la especie y la ausencia de mecanismos establecidos para la reincorporación de éstos al medio natural.

A partir de nuestro estudio sobre un total de siete osos hormigueros (Myrmecophaga tridactyla) adultos mantenidos en cautiverio intentamos evaluar: 1) si los individuos mantienen la capacidad de responder ante señales o signos de un depredador natural como el yaguareté (Panthera onca), aún siendo sometidos a largos períodos de cautividad; y 2) si la respuesta ante estímulos de distintos depredadores es específi$\mathrm{ca}$, siendo capaces de distinguir entre éstos y aquellos que no entablan un riesgo serio para los osos hormigueros. Con este objetivo se desarrollaron experimentos utilizando tanto estímulos químicos (heces de depredadores) como acústicos (llamadas del depredador), y caracterizando la respuesta observada.

\section{MATERIALES Y MÉTODOS}

El experimento fue desarrollado entre enero y julio de 2014. En total se trabajó con siete osos hormigueros adultos provenientes del Jardín Zoológico de Buenos Aires (JZBA; $\mathrm{n}=3$ ) y del Bioparque Temaikèn (BT; $n=4)$. Todos los individuos objeto de estudio provienen de la naturaleza pero pasaron la mayor parte de sus vidas en cautiverio desde muy temprana edad (entre 3 meses y 3 años; TABLA 1).

\section{Diseño experimental}

Los ensayos se llevaron a cabo entre las 09:00 y las 16:00 hs en días soleados y con poco viento. Cada ejemplar fue evaluado en forma independiente aislándolo del resto de los individuos. Para las experiencias de respuesta diferencial se utilizaron como tratamientos los sonidos y olores producidos por yaguareté, oveja (Ovis aries) y aguará guazú (Chrysocyon brachyurus). El primero es un depredador natural del oso hormiguero, teniendo una amplia simpatría histórica con el mismo. La oveja se utilizó como un control, siendo un animal totalmente inofensivo y alopátrico. El aguará guazú constituyó un segundo 
TABLA 1. Datos relevantes de los individuos de osos hormigueros utilizados en los experimentos de reconocimiento de depredadores. BT: Bioparque Temaikèn; JZBA: Jardín Zoológico de Buenos Aires.

\begin{tabular}{cccccc}
\hline $\begin{array}{c}\text { Número del } \\
\text { individuo }\end{array}$ & Ubicación actual & $\begin{array}{c}\text { Edad de puesta en } \\
\text { cautiverio }\end{array}$ & Edad (años) & $\begin{array}{c}\text { Tiempo en cau- } \\
\text { tiverio (años) }\end{array}$ & Procedencia \\
\hline 1 & JZBA & 1 año \pm 6 meses & 10 & 9 & Zoo. Roque Sáenz Peña, Chaco \\
2 & JZBA & 1 año \pm 6 meses & 9 & 8 & Zoo. Roque Sáenz Peña, Chaco \\
3 & JZBA & 2 años \pm 1 año & 19 & 17 & Desconocida \\
4 & BT & 5 meses \pm 2 meses & 4 & 3 & Estación de Fauna Autóctona, Salta \\
5 & BT & 2 años \pm 1 año & $9^{*}$ & 7 & Estación de Fauna Autóctona, Salta \\
6 & BT & 2 años \pm 1 año & $9^{*}$ & 7 & Estación de Fauna Autóctona, Salta \\
7 & BT & 2 años \pm 1 año & $9^{*}$ & 7 & Estación de Fauna Autóctona, Salta \\
\hline
\end{tabular}

* Se desconoce para estos individuos la edad exacta con la cual ingresaron al Bioparque, pero se presume que hubiera variado entre 1 y 3 años.

control, siendo éste una especie carnívora simpátrica pero que no entabla riesgo alguno para el oso hormiguero (Aragona \& Setz, 2001).

En cada ensayo se expuso al individuo focal a dos estímulos presentados en forma secuencial: uno químico-olfativo correspondiente a una muestra de materia fecal, y otro acústico correspondiente a una llamada, ambos de la especie tratamiento (yaguareté, oveja o aguará guazú). Como estímulo olfativo se utilizaron $100 \mathrm{~g}$ de heces colectadas el mismo día o el

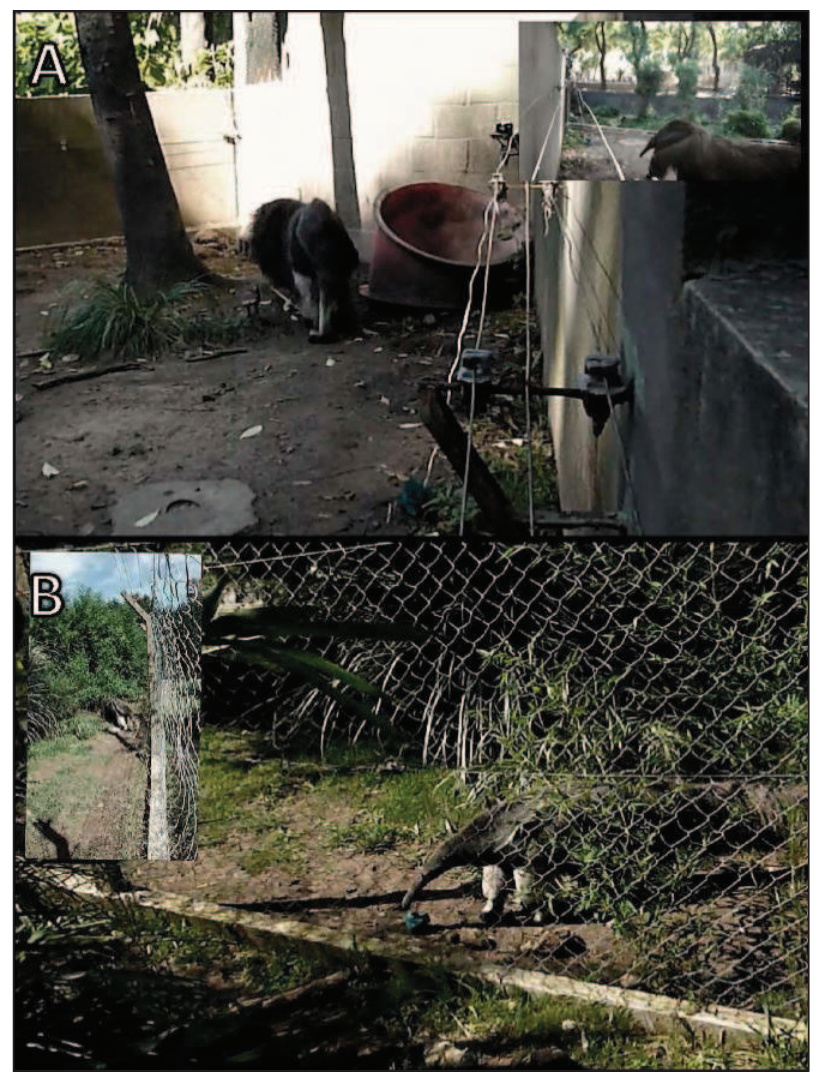

FIGURA 1. Realizado de experiencias con oso hormiguero (Myrmecophaga tridactyla) en A) Jardín Zoológico de Buenos Aires (JZBA) y B) Bioparque Temaikèn (BT), Argentina. día anterior al estudio, para que fueran lo más frescas posibles, maximizando así la intensidad de la señal y evitando que la degradación redujera el nivel de información de los compuestos químicos disponibles en la materia fecal (Wyatt, 2003), así como los metabolitos sulfurosos. Las heces se colocaron en una bolsa arpillera para prevenir el contacto directo del animal. Nunca se utilizó una misma muestra de heces para más de un individuo.

Los estímulos acústicos consistieron en la reproducción de llamados de las distintas especies. Dichos llamados fueron obtenidos de la red (Macaulay Library: aguará guazú y oveja, catálogos 176699 y 126289 [http://macaulaylibrary.org/]; YouTube y ARKive: yaguareté [https://www.youtube.com y http:/ / www.arkive.org]) y editados con el programa Adobe Soundbooth CS3 1.0 (Adobe Systems Incorporated, San José, California, USA). Se utilizaron vocalizaciones de al menos tres ejemplares para cada tratamiento. Si bien no se realizaron estudios exhaustivos de los repertorios vocales producidos por las distintas especies, se seleccionó de manera arbitraria un tipo de vocalización particular para cada caso y se tomaron sólo audios de alta fidelidad (relación sonido:ruido) para los mismos. Los audios fueron modificados de forma tal que tuvieran todos la misma duración y tasa de repetición. Así, las pistas obtenidas consistían en cuatro repeticiones de una vocalización de entre 1 y 1,5 s de duración, separadas entre sí por $3 \mathrm{~s}$ de silencio, teniendo una duración total de entre 13 y 15 s. Los archivos editados fueron guardados en formato WAV y reproducidos con un grabador portátil TASCAM DR-05 (TEAC Corporation, Tama, Tokio, Japón), conectado a un parlante potenciado NOGAnet (EMP Electronics S.A., Buenos Aires, Argentina). La intensidad de emisión fue medida a $1 \mathrm{~m}$ del dispositivo con un decibelímetro marca TES modelo 1350a (TES Electrical Electronic Corp., Taipei, Taiwan), controlando que se mantuviese en el rango de 82-86 dB en su máxima amplitud para los distintos estímulos. 
Para la realización del estudio se efectuó un tratamiento por individuo por semana, de forma tal de asegurarse que no existiera una habituación al estímulo por parte de los individuos. La secuencia de presentación de los estímulos correspondientes a cada tratamiento (yaguareté, oveja y aguará guazú) fue balanceado, eligiéndose al azar un orden determinado para cada individuo experimental.

En cada ensayo se ubicaron las heces dentro del recinto de oso hormiguero, en algún sitio donde fuesen fácilmente halladas por el individuo. El equipo de reproducción fue colocado en el límite del recinto o por fuera de éste a 2,5 m del estímulo olfativo y oculto al animal. La estructura (desniveles, vegetación para refugio y senderos de caminata) y el tamaño de los recintos tanto en el JZBA (trapecio de $187 \mathrm{~m}^{2}$ de superficie) como en el BT (rectángulo de $192 \mathrm{~m}^{2}$ de superficie) es suficiente como para permitir a los animales refugiarse, huir o retirarse del sector en que se dispusieron los estímulos.

Luego de montados los dispositivos, el individuo era liberado en el recinto externo (habiendo sido encerrado en el recinto interior la noche anterior como es de rutina). El estímulo acústico se desencadenó 2-3 min después de que el individuo testeado detectara el estímulo olfativo y fue reproducido sólo una vez, representando un incremento intenso e instantáneo en el riesgo de depredación para evitar cualquier posibilidad de habituación al estímulo. Luego de finalizada cada experiencia, se retiraron inmediatamente las heces, eliminando todo rastro posible del recinto. Todos los experimentos fueron filmados y las filmaciones fueron analizadas posteriormente en laboratorio.

\section{Análisis de datos}

La conducta del animal fue estudiada mediante el método focal continuo (Martin \& Bateson, 2007). Se reconocieron siete comportamientos de respuesta diferentes, los cuales fueron caracterizados basándose en las descripciones de Shaw et al. (1987) y Schmidt (2012). Estos comportamientos fueron:

- Piloerección: el animal detenido o caminando, eriza sus pelos de la zona superior-posterior del torso y la cola.

- Alarma olfativa: breve estado luego del estímulo olfativo, en que el animal avanza unos pasos, con su cabeza levantada por encima del eje longitudinal y el hocico hacia arriba.

- Alarma auditiva: el animal se posiciona rápidamente hacia el estímulo sonoro, se detiene y agacha repentinamente su cabeza con cada reproducción.

- Huida: luego del estímulo auditivo, el animal modifica su velocidad acelerando su caminata (levanta las extremidades anterior derecha $\mathrm{y}$ posterior izquierda al mismo tiempo y las desplaza hacia delante hasta colocarlas en el suelo; luego repite lo mismo pero con la extremidad anterior izquierda y la posterior derecha e inicia nuevamente) o corriendo (se impulsa con ambas extremidades anteriores mientras las posteriores avanzan en el aire, para luego alternarse), alejándose del mismo hasta perderse de vista por detrás de la vegetación o a través de ésta.

- Ejecución de giro: el animal se detiene, descansa su peso corporal en sus dos extremidades posteriores, arquea su columna hacia adentro y vuelve su cuerpo horizontalmente hacia la derecha o la izquierda, colocando a continuación los miembros anteriores en contacto con el suelo y reanudando su caminata en dirección opuesta.

- Investigación: el animal se encuentra de pie, mientras toca un objeto con su hocico y / o uno o ambos miembros anteriores.

- Alerta: breve estado luego de escuchar un sonido, en el cual el animal no se mueve interrumpiendo cualquier actividad que estaba llevando a cabo. Está de pie con las cuatro extremidades sobre el suelo (o una extremidad anterior semiflexionada hacia su región ventral), la cabeza en dirección a la fuente de sonido, y ojos abiertos.

Los comportamientos de Alerta e Investigación fueron los únicos que permitieron una cuantificación midiendo el tiempo dedicado a cada actividad. El resto de los comportamientos fueron analizados como una respuesta categórica (presencia-ausencia).

Para comparar los tiempos que los osos hormigueros dedicaron a la Investigación y a permanecer en Alerta durante la presentación de los estímulos, se utilizaron pruebas de Friedman de medidas repetidas. A fin de incluir las variables categóricas dentro de los análisis, se construyeron índices simples de respuesta que intentan reflejar la intensidad de las mismas ante el riesgo de depredación percibido. Este índice consistió en la suma de ocurrencias durante cada tratamiento, de cada una de las respuestas comportamentales descritas anteriormente. Los índices así confeccionados fueron comparados entre tratamientos con pruebas de Friedman de medidas repetidas. Los análisis estadísticos se realizaron mediante el software GenStat DE3 (VSN International Ltd., Hemel Hempstead, Hertfordshire, Inglaterra).

\section{RESULTADOS}

El comportamiento de los osos hormigueros frente a las señales olfativas no difirió entre tratamientos, siendo similar para heces de yaguareté, aguará guazú y oveja (prueba de Friedman, $\chi_{2}^{2}=0,5, P=0,78$ ). Aún cuando pudo observarse en mayor o menor medida el comportamiento de Ejecución de giro frente a los tres tratamientos, la conducta de Alarma olfativa sólo 
se detectó en dos individuos expuestos a aromas de yaguareté (Fig. 2).

La respuesta comportamental de los individuos frente al estímulo acústico medida por el índice confeccionado a partir de la presencia-ausencia de las distintas conductas, difirió entre tratamientos. Se observó una mayor cantidad de respuestas de los osos hormigueros ante llamados de yaguareté que ante estímulos de oveja y aguará guazú (prueba de Friedman, $\chi_{2}^{2}=7, \mathrm{P}=0,03$; Fig. 3). Cinco de los siete individuos tuvieron comportamientos que podrían considerarse antidepredatorios únicamente al ser tratados con su predador natural. Sin embargo, sólo dos de ellos mostraron el comportamiento de Huida (Fig. 4), lo cual es típico de la conducta de dicha especie en la naturaleza. Si bien un individuo mostró el comportamiento de Huida frente a las vocalizaciones tanto de yaguareté como de aguará guazú, en este último caso lo hizo acelerando notablemente su caminata mientras que para el primero respondió corriendo (el otro individuo sólo Huyó frente a yaguareté). Esto podría indicar aún más una capacidad de reconocer y responder de manera diferencial frente a los distintos estímulos. Lo mismo se ve reflejado en la mayor cantidad de comportamientos de Alarma auditiva, Ejecución de giro y Piloerección desplegados ante la señal acústica de yaguareté comparados a aquellos observados ante vocalizaciones de aguará guazú y oveja (Fig. 4).

Por otra parte, tanto el tiempo que los osos hormigueros invirtieron en el comportamiento de Investigación como en permanecer Alerta, no difirieron significativamente entre los distintos tratamientos (prueba de Friedman, $\chi_{2}^{2}=1,41, \mathrm{P}=0,49, \mathrm{y} \chi_{2}^{2}=1,61$, $\mathrm{P}=0,44$, para estímulo odorífero y acústico, respectivamente). En todos los casos las respuestas de los individuos duraron menos de $50 \mathrm{~s}$. La exclusión de aquellos dos individuos que desarrollaron la Huida inmediatamente después de la exposición a los estímulos acústicos y que por lo tanto no mostraron la conducta de Alerta, no modificó los resultados significativamente (prueba de Friedman, $\chi_{2}^{2}=3,89, \mathrm{P}=0,14$ ).

\section{Discusión}

En el presente trabajo pusimos a prueba la capacidad de reconocimiento de depredadores que poseen osos hormigueros mantenidos en cautividad por largos períodos de tiempo (3-17 años). En general se pudo observar que los mismos mantenían cierta habilidad de discriminación entre depredadores y no-depredadores, mostrando respuestas que fueron desde un incremento en el estado de Alarma hasta la Huida, en presencia de señales que pretendían simular la cercanía de un depredador. A pesar de ello, existió una alta variación tanto entre individuos como entre los estímulos presentados.

El análisis del ensayo utilizando heces de depredadores y especies inocuas demostró una falta de

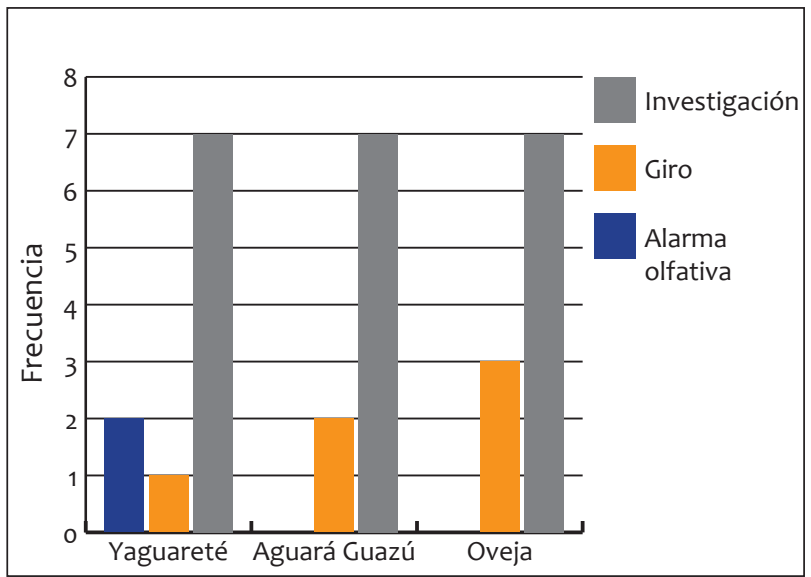

Figura 2. Frecuencia de ocurrencia para conductas de Investigación, Giro y Alarma olfativa en osos hormigueros (Myrmecophaga tridactyla) ante el estímulo olfativo de yaguareté, Panthera onca; aguará guazú, Chrysocyon brachyurus; y oveja, Ovis aries.

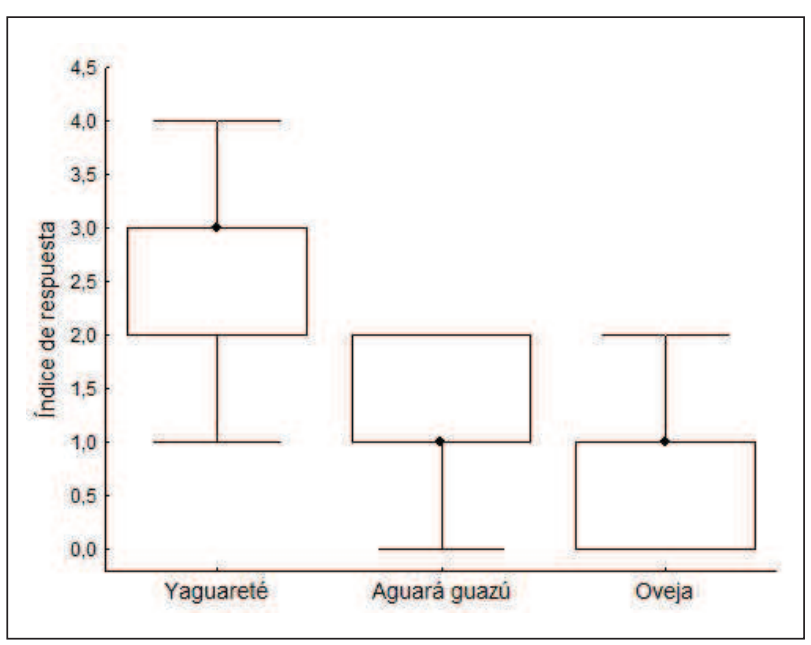

Figura 3. Variación del índice de respuesta en osos hormigueros (Myrmecophaga tridactyla) ante el estímulo acústico de yaguareté, Panthera onca; aguará guazú, Chrysocyon brachyurus; y oveja, Ovis aries. Se representan las medianas (puntos), los cuartiles del 25 y 75\% (caja) y el rango (líneas).

diferencias en las respuestas de los osos hormigueros ante los diferentes tratamientos. Sin embargo, se observó una tendencia no significativa a incrementar el tiempo de Investigación sobre las heces cuando éstas eran de oveja en comparación con las de depredadores y la presencia única del comportamiento de Alarma olfativa cuando eran de yaguareté. Es posible entonces que esto refleje un comportamiento de evitación y una respuesta específica frente a los depredadores y sus olores. A pesar de ello, esta respuesta fue débil en los osos hormigueros y estadísticamente no significativa. La falta de diferencias en la conducta puede ser consecuencia no solamente del bajo tamaño de muestra. Las claves olfativas derivadas de heces $u$ orina pueden constituir señales poco confiables en cuanto a que las mismas persisten en el ambiente por períodos largos luego de que el animal que las 


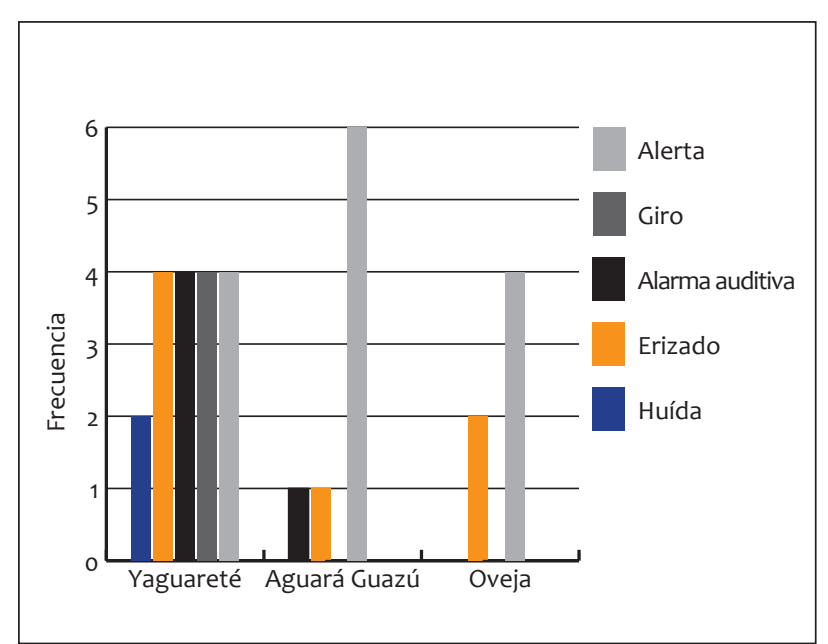

FIGURA 4. Frecuencia de ocurrencia para conductas de Alerta, Giro y Alarma auditiva, Piloerección (erizado) y Huida en osos hormigueros (Myrmecophaga tridactyla) ante el estímulo acústico de yaguareté, Panthera onca; aguará guazú, Chrysocyon brachyurus; y oveja, Ovis aries.

produjera hubiera abandonado el sitio (Bradbury \& Vehrencamp, 2011). Esto podría hacer que la selección actúe contra el uso de señales olfativas como señales confiables del riesgo de depredación.

Las respuestas de los osos hormigueros ante los estímulos acústicos fueron más claras que aquellas dadas frente a estímulos olfativos. Si bien la duración del comportamiento de Alerta luego de que se reprodujeran los distintos audios no mostró diferencias significativas entre tratamientos, se observó una tendencia a que el tiempo de dicha conducta fuera mayor frente a los sonidos emitidos por depredadores. Aún más importante, se detectó que ante los llamados de yaguareté los osos hormigueros respondieron incrementando aquellos comportamientos que reflejan un estado de alarma y evitación. Usualmente los individuos al escuchar esta vocalización se detenían y direccionaban su cabeza hacia el sitio de dónde provenía el llamado (Alerta), agudizando probablemente sus sentidos, acompañado esta vez de un momento de estrés claramente representado por una Alarma auditiva y subsiguiente evitación (Ejecución de giro o Huida) del sitio. Tales respuestas fueron específicas y no se detectaron al exponer a los animales a estímulos de especies no-depredadoras.

La variación individual en las respuestas observadas puede reflejar una gradual desensibilización al estímulo. En tal sentido, se ha sugerido que los animales mantenidos en cautiverio carecen de las habilidades para sobrevivir en estado salvaje (Swaisgood et al., 2001). Los individuos en esta situación pueden perder la sensibilidad a los estímulos, no sólo como se mencionó anteriormente debido a los costos del mantenimiento del comportamiento antidepredatorio, sino también debido a las posibles perturbaciones que adiciona el ambiente de cautividad (como ruido de fondo, velocidad del viento, etc.) o a la seguridad percibida dentro del refugio, modificando así la percepción de los estímulos (Lima \& Dill, 1990). En el caso de los individuos del JZBA, el recinto de los osos hormigueros se encuentra en la cercanía de distintas especies de felinos, por lo tanto es de esperarse que reciban estímulos olfativos y auditivos a diario que generen un acostumbramiento y gradual degradación de la respuesta. Esto no sucede en el BT.

Los resultados preliminares obtenidos parecen entonces confirmar que los osos hormigueros pueden retener cierta capacidad de reconocimiento de sus depredadores a pesar de ser mantenidos en cautiverio por tiempos prolongados. Dada la corta edad a la cual los animales experimentales fueron capturados y la baja o nula densidad de yaguaretés presentes en las regiones de donde provinieron estos osos hormigueros, podemos suponer la ausencia de experiencias previas o contacto alguno de los sujetos de investigación con sus depredadores en la naturaleza. De esta forma, la probabilidad de que existiera un aprendizaje de evitación en los mismos es extremadamente baja, y aún cuando la hubieran tenido, el tiempo que han permanecido en cautiverio podría ofrecer las condiciones para que las conductas de reconocimiento y evitación de depredadores se perdieran (ver Coss, 1999). Es posible entonces que dicha respuesta sea independiente de la experiencia que tengan con ellos, y se deba únicamente al contacto evolutivo.

Dicho hallazgo es de suma importancia a la hora de definir estrategias, por ejemplo para la reintroducción de animales criados en cautiverio y la reconstrucción de ecosistemas que incluyan a los depredadores superiores. La reinserción de individuos aislados por largo tiempo de su medio natural, usualmente falla debido a problemas comportamentales de los animales liberados, especialmente aquellos relacionados a su conducta de alimentación, evitación de depredadores y conducta social (Snyder et al., 1996). Así, estudios como el presente han sido fuertemente recomendados para cualquier caso de reintroducción y restauración de hábitats (ver Curio, 1996; Caro, 2005), ya que ataca a algunas de las críticas más fuertes que se realizan a la conservación ex situ.

Otro aspecto importante que se desprende de este trabajo se relaciona a la respuesta observada en los osos hormigueros frente a los estímulos olfativos y acústicos de depredadores. La evitación de los mismos, ya sea cambiando de dirección o huyendo del sitio, sugieren que dichas señales pueden tener también una interesante aplicación como "repelentes" para la especie presa, protegiendo así a los osos hormigueros de zonas de peligro como rutas y áreas agrícolas o residenciales. Sin embargo, serían necesarios más estudios para determinar la eficacia global de este tipo de estímulos, ya que no todos los individuos han mostrado este comportamiento de evitación, ni una amplia duración en el tiempo. 


\section{AGRADECIMIENTOS}

Agradecemos a todas las personas e instituciones que cedieron su tiempo para la realización de las experiencias tanto en el Jardín Zoológico de Buenos Aires como en el Bioparque Temaikèn (Lic. Giovanna Donini, Lic. Paula González, Lic. Florencia Presa, Dr. Andrés Palmerio y Cuidadores de ambas instituciones).

\section{REFERENCIAS}

Anson, J. \& C. Dickman. 2013. Behavioral responses of native prey to disparate predators: naivete and predator recognition. Oecologia 171: 367-377.

Aragona, M, \& E. Setz. 2001. Diet of the maned wolf, Chrysocyon brachyurus (Mammalia: Canidae), during wet and dry seasons at Ibitipoca State Park, Brazil. Journal of Zoology 254: 131-136.

Beck, B., D. Kleiman, J. Dietz, I. Castro, C. Carvalho, A. Martins \& B. Rettberg-Beck. 1991. Losses and reproduction in reintroduced golden lion tamarins Leontopithecus rosalia. Jersey Wildlife Preservation Trust 27: 50-61.

Berger, J., J. Swenson \& I. Persson. 2001. Recolonizing carnivores and naive prey: conservation lessons from Pleistocene extinctions. Science 291: 1036-1039.

Blumstein, D. 2002. Moving to suburbia: ontogenetic and evolutionary consequences of life on predator-free islands. Journal of Biogeography 29: 685-692.

Blumstein, D., A. Bitton \& J. DaVeiga. 2006. How does the presence of predators influence the persistence of antipredator behavior? Journal of Theoretical Biology 239: 460-468.

Blumstein, D., J. Daniel, A. Griffin \& C. Evans. 2000. Insular tammar wallabies (Macropus eugenii) respond to visual but not acoustic cues from predators. Behavioral Ecology 11: 528-535.

Bradbury, J. \& S. Vehrencamp. 2011. Principles of animal communication. Sinauer Associates, Sunderland. 697 pp.

Brown, J. 1999. Vigilance, patch use, and habitat selection: foraging under predation risk. Evolutionary Ecology Research 1: 49-71.

Brown, G. \& D. Chivers. 2005. Learning as an adaptive response to predation. Pp. 34-54 in: Ecology of predator-prey interactions (P. Barbosa \& I. Castellanos, eds.). Oxford University Press, New York.

Caro, T. 2005. Antipredator defenses in birds and mammals. University of Chicago Press, Chicago. $592 \mathrm{pp}$.
Coss, R. 1999. Effects of relaxed natural selection on the evolution of behavior. Pp. 180-208 in: Geographic variation in behavior: perspectives on evolutionary mechanisms (S. A. Foster \& J. A. Endler, eds.). Oxford University Press, New York.

Curio, E. 1996. Conservation needs ethology. Trends in Ecology and Evolution 11: 260-263.

Endler, J. 1991. Interaction between predators and prey. Pp. 169-196 in: Behavioural ecology: an evolutionary approach (J. R. Krebs \& N. B. Davis, eds.). Blackwell Scientific Publications, Oxford.

Fonseca, G. A. B. \& J. Aguiar. 2004. The 2004 Edentate species assessment workshop. Edentata 6: 1-26.

Hollén, L. \& M. Manser. 2007. Persistence of alarmcall behaviour in the absence of predators: a comparison between wild and captive-born meerkats (Suricata suricatta). Ethology 113: 1038-1047.

IUCN. 2014. The IUCN Red List of Threatened Species 2014. International Union for Conservation of Nature and Natural Resources. $<$ http://www.iucnredlist.org/>. Consultada 2 de octubre de 2014.

Lima, S. \& L. Dill. 1990. Behavioral decisions made under the risk of predation - a review and prospectus. Canadian Journal of Zoology 68: 619-640.

Magurran, A. 1999. The causes and consequences of geographic variation in antipredator behavior: perspectives from fish populations. Pp. 139-163 in: Geographic variation in behavior: perspectives on evolutionary mechanisms (S. A. Foster \& J. A. Endler, eds.). Oxford University Press, New York.

Martin, P. \& P. Bateson. 2007. Measuring behaviour: an introductory guide. Cambridge University Press, Cambridge. 186 pp.

Miller, B., D. Biggins, L. Hanebury \& A. Vargas. 1994. Reintroduction of the black-footed ferret (Mustela nigripes). Pp. 455-464 in: Creative conservation: interactive management of wild and captive animals (P. J. Olney, G. M. Mace \& A. T. C. Feistner, eds.). Chapman and Hall, London.

Monclús, R., H. Rödel, D. Von Holst \& J. De Miguel. 2005. Behavioural and physiological responses of naïve European rabbits to predator odour. Animal Behaviour 70: 753-761.

Pérez Jimenez, I. 2006. Plan de recuperación de oso hormiguero gigante en los esteros de Iberá, Corrientes (2006-2010). Dirección de Recursos Naturales y The Conservation Land Trust, Corrientes. 
Schmidt, T. 2012. Ethogram of the giant anteater (Myrmecophaga tridactyla) in captivity: an experience in the Temaikèn Foundation. Edentata 13: 38-48.

Shaw, J., J. Machado-Neto \& T. Carter. 1987. Behavior of free-living giant anteaters (Myrmecophaga tridactyla). Biotropica 19: 255-259.

Short, J., S. Bradshaw, J. Giles, R. Prince \& G. Wilson. 1992. Reintroduction of macropods (Marsupialia: Macropodoidea) in Australia - a review. Biological Conservation 62: 189-204.

Snyder, N., S. Derrickson, S. Beissinger, J. Wiley, T. Smith, W. Toone \& B. Miller. 1996. Limitations of captive breeding in endangered species recovery. Conservation Biology 10: 338-348.

Superina, M., A. M. Abba \& S. F. Vizcaíno. 2012. Orden Pilosa. Pp. 59-60 in: Libro Rojo de los mamíferos de Argentina (R. A. Ojeda, V. Chillo \& G. Díaz Isenrath, eds.). SAREM, Mendoza, Argentina.

Swaisgood, R. R, A. M. White, X. Zhou, H. Zhang, G. Zhang, R. Wei, V. J. Hare, E. M. Tepper \& D. G. Lindburg. 2001. A quantitative assessment of the efficacy of an environmental enrichment programme for giant pandas. Animal Behaviour 61: 447-457.

Wetzel, R. 1982. Order Edentata-Xenarthra. Pp. 52-57 in: Mammal species of the world (J. H. Honacki, K. E. Kinnan \& J. W. Koeppl, eds.). Allen Press, Lawrence, Kansas.

Wyatt, T. 2003. Pheromones and animal behaviour: Communication by smell and taste. Cambridge University Press, Cambridge. 391 pp.

Recibido: 3 de octubre de 2014; Aceptado: 3 de diciembre de 2014 